\section{J. Escarrabill}

Master Plan for Respiratory Diseases - PDMAR - Department of Health, Institut d'Estudis de la Salut, Barcelona, Spain

\section{Correspondence}

J. Escarrabill

Institut d'Estudis de la Salut Cl Roc Bornat, 81-95 1st floor 08005 Barcelona

Spain

jescarrabill@gencat.cat

\title{
Practical issues about sleep disorders and the diagnosis of chronic cough
}

This issue of Breathe has two main topics. On the one hand, three articles deal with sleep disorders and, on the other hand, one article discusses important clinical issues in chronic cough.

Sleep disorders have a significant clinical impact, with both health and socially relevant importance. The high number of patients suffering from these problems (in some cases with lifethreatening symptoms) and the burden on healthcare organisations related to the impact of long-term cost justify the interest in sleep respiratory disorders. The social impact relates to traffic, domestic and workplace accidents that are directly related to sleep disorders.

Continuous positive airway pressure (CPAP) is one of the fundamental bases for sleep disorder treatment in adults. Closing the circle of detection, diagnosis, degree of pressure and compliance is sometimes very complex. The variability across different European countries probably responds to the different solutions adopted in each country to address the four elements of that circle. The variability observed in the number of patients treated, the accessibility (waiting lists) and the followup is very important. The health model has a definitive influence on the service. In a liberal model, the existence of waiting lists is hardly acceptable, while in a model based on the National Health Service or social security, the lack of health coverage for groups of citizens it is not acceptable.

One of the challenges of most health systems is to efficiently organise the follow-up of patients with CPAP. A follow-up based on specialised centres (mainly hospitals) is not sustainable. The review in this issue by Martínez-García and Catalán Serra [1] discusses the role of general practitioners in the care of patients with CPAP. An initial objective should be an improvement in the identification of sleep disorders in firstline care. General practitioners routinely ask patients about allergies, consumption of alcohol or physical activity, but it is not routine to ask about the characteristics and quality of sleep.

Strengthening the role of general practitioners in the management of sleep disorders is very important. This process requires continuous education, strategies for detecting cases and referral protocols that prioritise the transfer to specialised centres according to severity and defining long-term monitoring.

In some cases, patients do not tolerate CPAP. In this issue, VANDERVEKEN and HOEKEMA [2] summarise very well the problems of treatment with CPAP related to the interface (interface intolerance, excessive pressure and difficulty getting to sleep) or the direct complications of treatment. Oral appliance therapies (mainly mandibular repositioning appliances (MRAs)) may play a role in cases of intolerance to CPAP or, even, as the authors suggest, in patients that refuse CPAP therapy.

It is very important to note that the use of these alternatives to CPAP require an adjustment period and they should be prescribed in a personalised manner; therefore, a period of titration of the MRAs is essential for the resolution of symptoms with minimal discomfort and sideeffects.

In some cases, patients need surgical procedures at nasal, retropalatal or retrolingual level. In all cases, doctors emphasise the 
benefits of a multidisciplinary approach in order to choose the best adapted solution to the patients needs.

Cough, along with dyspnoea, is one of the most characteristic manifestations of respiratory diseases, although, sometimes, the cause may be extrapulmonary. The explosive expiration that characterises cough is basically a defense mechanism that, most often, it is due to a benign cause and is selflimiting.

Accurate diagnosis of the cause of cough is sometimes very difficult. Precisely for this reason, it is very important that a systematic approach is taken to diagnosis of cough. In most cases, acute cough usually lasts 3 weeks and is only exceptionally longer than 8 weeks. The clinical manifestations are very important to determine if the cough is acute or chronic, if associated with a respiratory infection or if seasonal changes present, for example.

However, the challenge is the diagnosis of chronic cough, especially in patients with normal chest radiograph and basic lung function without abnormalities. In this issue, KARDOS [3] points out, quite rightly, possible errors that should be avoided in the diagnosis of chronic cough: use of angiotensin-converting enzyme inhibitors, the trivialisation of cough in patients with chronic obstructive pulmonary disease and inadequate consideration of extrapulmonary causes (ear, nose and throat, gastric, neurological, and cardiac).

References

1. Martínez-García MA, Catalán Serra P. Is sleep apnoea a specialist condition? The role of general practicioners. Breathe 2010; 7: 42-53.

2. Vanderveken OM, Hoekema A. How to treat patients that do not tolerate continuous positive airway pressure. Breathe 2010; 7: 54-65.

3. Kardos P. Management of cough in adults. Breathe 2010; 7: 20-32. 